\title{
Sopa instantânea com inclusão de farinhas de peixes
}

\author{
Instant noodle with fish meal inclusion
}

Sopa instantánea con inclusión de harina de pescado

Maria Luiza Rodrigues de Souza ORCID: https://orcid.org/0000-0001-5643-0841 Universidade Estadual de Maringá, Brasil E-mail: mlrsouza@uem.br

Allan Vinnícius Urbich

ORCID: https://orcid.org/0000-0002-6228-4956 Universidade Estadual de Maringá, Brasil E-mail: allan_vinnicius@hotmail.com

Bianca de Oliveira Müller

ORCID: https://orcid.org/0000-0003-4192-3867 Universidade Estadual de Maringá, Brasil E-mail: biancamuller1996@gmail.com

Melina Franco Coradini

ORCID: https://orcid.org/0000-0001-7351-0237 Universidade Estadual de Maringá, Brasil

E-mail: melinacoradini@gmail.com

Gislaine Gonçalves Oliveira

ORCID: https://orcid.org/0000-0002-7819-3493 Universidade Estadual de Maringá, Brasil E-mail: gislaine_oliveira14@hotmail.com

Marcos Antonio Matiucci

ORCID: https://orcid.org/0000-0003-2053-2672 Universidade Estadual de Maringá, Brasil

E-mail:m.matiucci@hotmail.com

Sabrina Campos Sbaraini

ORCID: https://orcid.org/0000-0002-7165-6563 Universidade Estadual de Maringá, Brasil

E-mail: sabrinacsbaraini@gmail.com

Gustavo Lazarini Martins

ORCID: https://orcid.org/0000-0003-2771-3492 Universidade Estadual de Maringá, Brasil E-mail: gustavolazarinim@gmail.com

Andresa Carla Feihrmann

ORCID: https://orcid.org/0000-0003-2389-0467 Universidade Estadual de Maringá, Brasil E-mail: andresafeihrmann@gmail.com

Elenice Souza dos Reis Goes

ORCID: https://orcid.org/0000-0003-2437-4800 Universidade Federal da Grande Dourados, Brasil E-mail: elenicegoes@ufgd.edu.br

\begin{abstract}
Resumo
Esse trabalhado teve como objetivo a elaboração de uma sopa instantânea com inclusão de farinha de peixes, avaliando a qualidade nutricional, microbiológica e sensorial. Foram desenvolvidas quatro sopas instantâneas, contendo diferentes farinhas de peixes (tilápia, tilápia defumada, tilápia defumada com salmão, e tilápia defumada com atum). Após a elaboração das farinhas estas foram adicionadas aos ingredientes da sopa, homogeneizados, embalados e analisados quanto a sua composição nutricional e microbiológica, depois preparada a sopa com adição de água quente para a análise sensorial. Todas as misturas das sopas e farinhas de peixe estavam dentro dos padrões microbiológicos para consumo. A mistura da sopa com inclusão de farinha de tilápia apresentou maior teor de umidade e menor valor calórico. As sopas com farinha de tilápia defumada e de tilápia defumada com salmão apresentaram maiores teores de lipídeos $(6,90 \%$ e $6,89 \%$, respectivamente) e de tilápia defumada com atum, o menor teor de cinzas. Não houve diferença para proteína e carboidratos, assim como, para a cor e para a textura das sopas. A mistura de sopa instantânea com farinha de tilápia defumada com atum teve pior aceitação no atributo aroma. O sabor, a impressão global e a intenção de compra foram piores para a sopa com inclusão de farinha defumada com atum. Conclui-se que as misturas de sopas instantâneas enriquecidas com farinha de peixe são nutritivas, com melhor aceitação para as sopas com farinha de tilápia, de tilápia defumada e tilápia defumada com salmão.
\end{abstract}

Palavras-chave: Carcaça de atum; Espinhaço de salmão; Resíduos de filetagem de tilápia. 


\begin{abstract}
This work aimed to prepare an instant noodle with the inclusion of fish meal, evaluating the nutritional, microbiological and sensory quality. Four instant soups were developed, containing different fish flours (tilapia, smoked tilapia, smoked tilapia with salmon, and smoked tilapia with tuna). After the preparation of the flours, these were added to the soup ingredients, homogenized, packaged and analyzed for their nutritional and microbiological composition, then the soup was prepared with the addition of hot water for sensory analysis. All soup and fish meal mixtures were within microbiological standards for consumption. The soup mixture with inclusion of tilapia flour had higher moisture content and lower caloric value. Soups with smoked tilapia and smoked tilapia with salmon flour had the highest levels of lipids $(6.90 \%$ and $6.89 \%$, respectively) and smoked tilapia with tuna had the lowest ash content. There was no difference for protein and carbohydrates, as well as for the color and texture of soups. The mixture of instant soup with smoked tilapia flour with tuna had the worst acceptance in the aroma attribute. The taste, overall impression and purchase intent were worse for the soup with the inclusion of smoked tuna flour. It was concluded that instant soup mixtures enriched with fish meal are nutritious, with better acceptance for soups with tilapia, smoked tilapia and smoked tilapia with salmon meal.
\end{abstract}

Keywords: Tuna carcass; Salmon backbone; Tilapia filleting residues.

\title{
Resumen
}

Este trabajo tuvo como objetivo preparar una sopa instantánea con la inclusión de harina de pescado, evaluando la calidad nutricional, microbiológica y sensorial. Se elaboraron cuatro sopas instantáneas, conteniendo diferentes harinas de pescado (tilapia, tilapia ahumada, tilapia ahumada con salmón y tilapia ahumada con atún). Después de la preparación de las harinas, estas se agregaron a los ingredientes de la sopa, se homogeneizaron, empacaron y analizaron su composición nutricional y microbiológica, luego se preparó la sopa con la adición de agua caliente para análisis sensorial. Todas las mezclas de sopa y harina de pescado estaban dentro de los estándares microbiológicos para el consumo. La mezcla de sopa con inclusión de harina de tilapia tuvo mayor contenido de humedad y menor valor calórico. Las sopas con tilapia ahumada y tilapia ahumada con harina de salmón tuvieron los niveles más altos de lípidos $(6,90 \%$ y $6,89 \%$, respectivamente) y la tilapia ahumada con atún tuvo el menor contenido de cenizas. No hubo diferencia para las proteínas y los carbohidratos, así como para el color y la textura de las sopas. La mezcla de sopa instantánea con harina de tilapia ahumada con atún tuvo la peor aceptación en el atributo de aroma. El sabor, la impresión general y la intención de compra fueron peores para la sopa con la inclusión de harina de atún ahumado. Se concluyó que las mezclas de sopas instantáneas enriquecidas con harina de pescado son nutritivas, con mejor aceptación para las sopas con tilapia, tilapia ahumada y tilapia ahumada con harina de salmón.

Palabras clave: Canal de atún; Espinazo de salmón; Residuos de fileteado de tilapia.

\section{Introdução}

A sopa é um líquido preparado a partir de vegetais, peixe ou carne com água, suco ou caldo e alguns agentes espessantes, e se enquadra na categoria heterogênea de alimentos (Islam et al., 2018). A sopa instantânea é quase pronta para comer e leva menos tempo para cozinhar (Karthika et al., 2016), sendo uma comida muito popular na sociedade moderna (Islam et al., 2018). Assim, existe uma grande demanda por misturas para sopas instantâneas no mercado global (Li \& Savage, 2015).

O consumo de sopas instantâneas de peixe pode fornecer uma quantidade considerável de proteínas à dieta (Sudarsan et al., 2017), uma vez que o pescado é boa fonte de proteínas de alta qualidade, assim como de vitaminas e minerais (Tilami \& Sampels, 2018). A inclusão de pescado em sopas instantâneas pode ser feita através de farinhas obtidas com o aproveitamento de resíduos do processamento, podendo agregar valor aos subprodutos. Sabe-se que muitos resíduos do processamento têm grande valor nutritivo, contendo altos níveis de proteínas, cinzas e lipídios (Monteiro et al., 2014).

O desenvolvimento de sopas instantâneas a partir de farinhas de peixes foi realizado por alguns autores (Monteiro et al., 2014; Islam et al., 2018), porém novos estudos são necessários para caracterizar este produto e torná-lo viavel para produção e comercialização.

Nesse sentido, a produção de farinhas de peixes a partir de resíduos da filetagem já foi bem documentada (Souza et al., 2017). A defumação das carcaças de peixes antes da produção das farinhas pode conferir o sabor de defumado ao produto em que for incluso, trazendo um diferencial tecnológico. Sabe-se que defumação é amplamente utilizada para melhorar o sabor, a cor e o aroma dos alimentos (Škaljac et al., 2014). Farinhas de carcaças defumadas de tilápia do Nilo foram desenvolvidas por Godoy et al. (2013), e tiveram boa aceitação sensorial em produtos como: palito de cebola (Coradini et al., 2015), snacks (Justen 
et al., 2017) e patês (Matiucci et al., 2019).

Assim, o objetivo do trabalho foi produzir e caracterizar farinhas a partir de resíduo de filetagem de peixes e elaborar sopas instantâneas com farinhaa de diferentes espécies de peixes (tilápia, salmão e atum) e avaliar sua composição nutricional, qualidade microbiológica e atributos sensoriais.

\section{Metodologia}

\section{Elaboração da farinha a partir de carcaças de tilápia, salmão e atum.}

A produção das farinhas de peixe foi realizada no Laboratório de Tecnologia de Pescado da Fazenda Experimental de

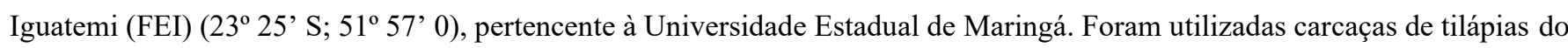
Nilo (Oreochromis niloticus) provenientes da Smart Fish (Rolândia-PR), carcaças de salmão (Salmo salar) da Empresa Tomita \& Tomita Ltda (Maringá-PR) e atum (Tunnus sp ) da Empresa Gomes da Costa (Itajaí-SC).

Foram retiradas das carcaças (espinhaço sem os filés) as nadadeiras e cabeças da tilápia e do salmão, no atum foram utilizadas a extremidade final do tronco, próximo a cauda, sem nadadeiras. Para a elaboração das farinhas (Figura 1), estas diferentes matérias- primas foram separadamente lavadas, pesadas e submetidas ao cozimento em panela de pressão com antioxidante $(\mathrm{BHT}=0,1 \%)$ por 60 minutos.

Para obtenção das farinhas de tilápia defumada as carcaças foram imersas em salmoura a 20\% (2:1 - volume da salmoura/peso) com extrato de alecrim, posteriormante drenadas e submetidas à secagem em estufa de circulação forçada de ar, por 30 minutos a $50^{\circ} \mathrm{C}$. Em seguida, as carcaças foram submetidas à câmara de defumação com adição de fumaça sendo utilizada para isso a serragem de eucalipto rosa (Eucalyptus globulus). A temperatura do defumador foi mantida entre $60-70^{\circ} \mathrm{C}$ com o auxílio de gás de cozinha.

Em seguida, tanto as carcaças cozidas quanto a defumada foram prensadas separadamente em prensa hidráulica com capacidade de 10 toneladas e moídas. Após a moagem, o produto foi desidratado em estufa de circulação de ar forçada a $60^{\circ} \mathrm{C}$, por $24 \mathrm{~h}$, moídas em moinho tipo faca (Willye - modelo TE-650), embaladas a vácuo e congeladas a $-18^{\circ} \mathrm{C}$ para aquelas amostras que foram submetidas à análises de composição centesimal, já para a análise microbiológica, as amostras foram armazenadas sob refrigeração $\left(5\right.$ a $\left.7^{\circ} \mathrm{C}\right)$ até a realização das análises.

Para a obtenção da farinha de salmão foram incluídos $20 \%$ do produto desidratado de salmão no produto desidratado de tilápia $(80 \%)$. Para a obtenção das farinhas de atum foram incluídos $20 \%$ do produto desidratado de atum no produto desidratado de tilápia (80\%). 
Figura 1. Elaboração de farinhas.
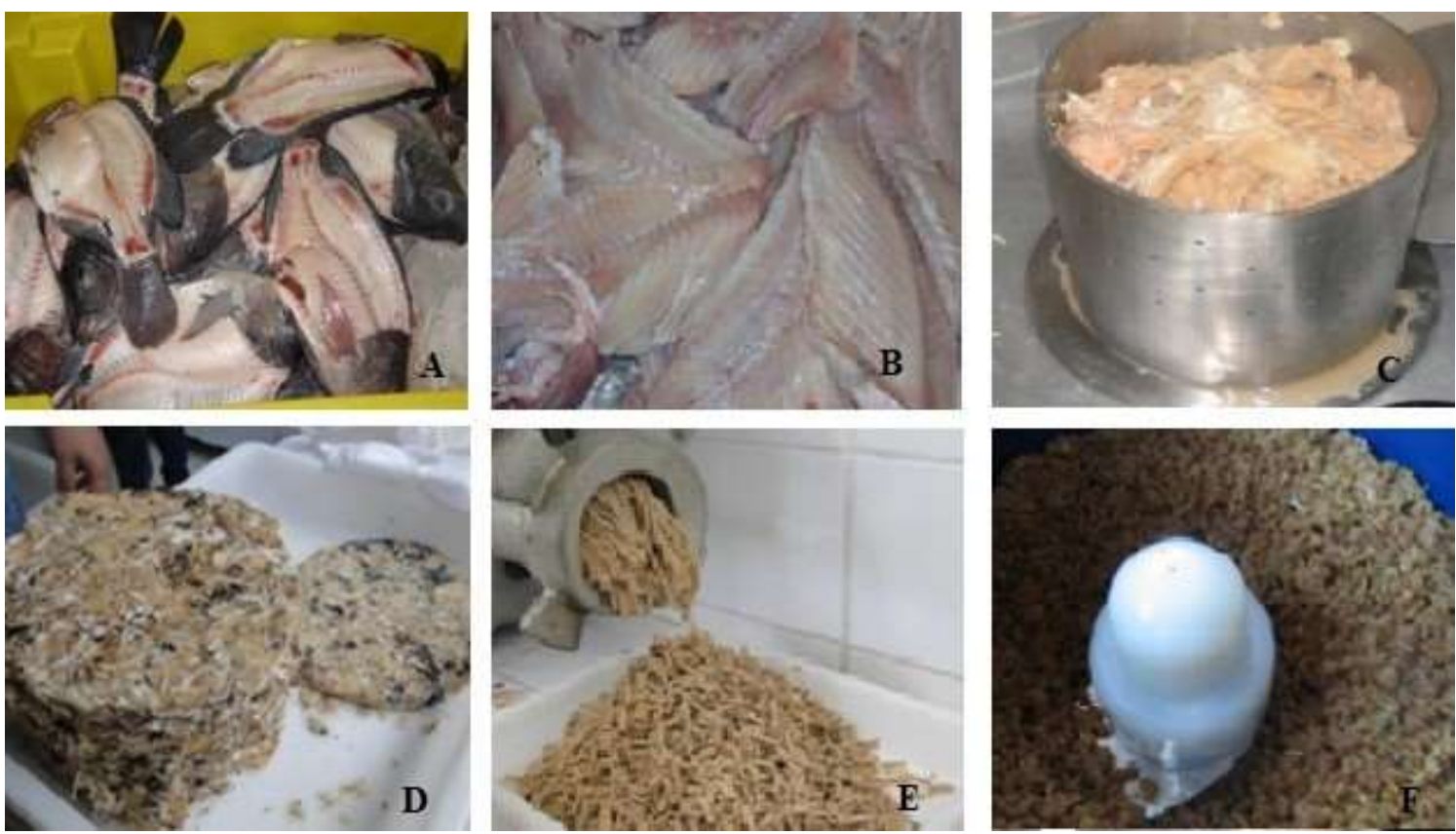

Fonte: Autores.

$\mathrm{Na}$ imagem "A” é possível observar as carcaças com cabeça e nadadeira, após o processo de filetagem, já a imagem "B" têm-se as carcaças sem cabeça e nadadeiras, prontas para o cozimento, a imagem "C" observa-se carcaças de salmão acondicionadas para o processo de prensagem, enquanto a imagem " $\mathrm{D}$ ” apresenta as carcaças de salmão após a prensagem, na imagem "E" 'd demonstrado a moagem das carcaças para posterior desidratação, sendo a imagem "F" o processo de trituração após desidratadas.

\section{Elaboração da mistura para sopa instantânea com inclusão de farinhas de peixes}

As sopas instantâneas foram preparadas no Laboratório de Tecnologia de Pescado na Fazenda Experimental de Iguatemi (FEI), da Universidade Estadual de Maringá. Para sua elaboração foi utilizada uma formulação padrão, cujos ingredienetes e proporções estão especificadas na Tabela 1. Os tratamentos foram constituídos por: Trat. 1= sopa com inclusão de farinha de tilápia (SFT), Trat. 2= sopa com farinha de tilápia defumada (SFTD), Trat. 3= sopa com farinha de tilápia defumada com salmão (SFTDS) e Trat. 4= sopa com farinha de tilápia defumada com atum (SFTDA). 
Research, Society and Development, v. 10, n. 8, e35910817247, 2021

(CC BY 4.0) | ISSN 2525-3409 | DOI: http://dx.doi.org/10.33448/rsd-v10i8.17247

Tabela 1. Ingredientes utilizados para elaboração da mistura de sopa instantânea.

\begin{tabular}{lc}
\hline \multicolumn{1}{c}{ Ingredientes } & Quantidades \\
\hline Farinha de Tilápia & $36 \mathrm{~g}$ (Trat $1=$ SFT) \\
Farinha de Tilápia Defumada & $36 \mathrm{~g}$ (Trat $2=$ SFTD) \\
Farinha de tilápia defumada e salmão & $36 \mathrm{~g}$ (Trat $3=$ SFTDS) \\
Farinha de tilápia defumada e atum & $36 \mathrm{~g}$ (Trat $4=$ SFTDA) \\
Amido de milho & $80 \mathrm{~g}$ \\
Aveia & $180 \mathrm{~g}$ \\
Farinha de mandioca & $180 \mathrm{~g}$ \\
Glutamato monossódico & $34 \mathrm{~g}$ \\
Goma Guar & $35 \mathrm{~g}$ \\
Leite em pó & $67 \mathrm{~g}$ \\
Sal & $18 \mathrm{~g}$ \\
Creme de leite & $67 \mathrm{~g}$ \\
Cebola em floco & $25 \mathrm{~g}$ \\
Alho-poró & $25 \mathrm{~g}$ \\
Cebolinha & $25 \mathrm{~g}$ \\
Salsinha & $25 \mathrm{~g}$ \\
Extrato de levedura & $52 \mathrm{~g}$ \\
Açafrão & $11 \mathrm{~g}$ \\
\hline &
\end{tabular}

Fonte: Autores.

Para a preparação das misturas as farinhas foram preparadas separadamente. Os ingredientes foram pesados de acordo com a formulação de cada tratamento (Figura 2), homogeneizados, acondicionadas em embalagens plásticas previamente identificadas de acordo com a farinha de peixe utilizado na elaboração.

Figura 2. Ingredientes para mistura de sopa instantânea com farinha de peixe.

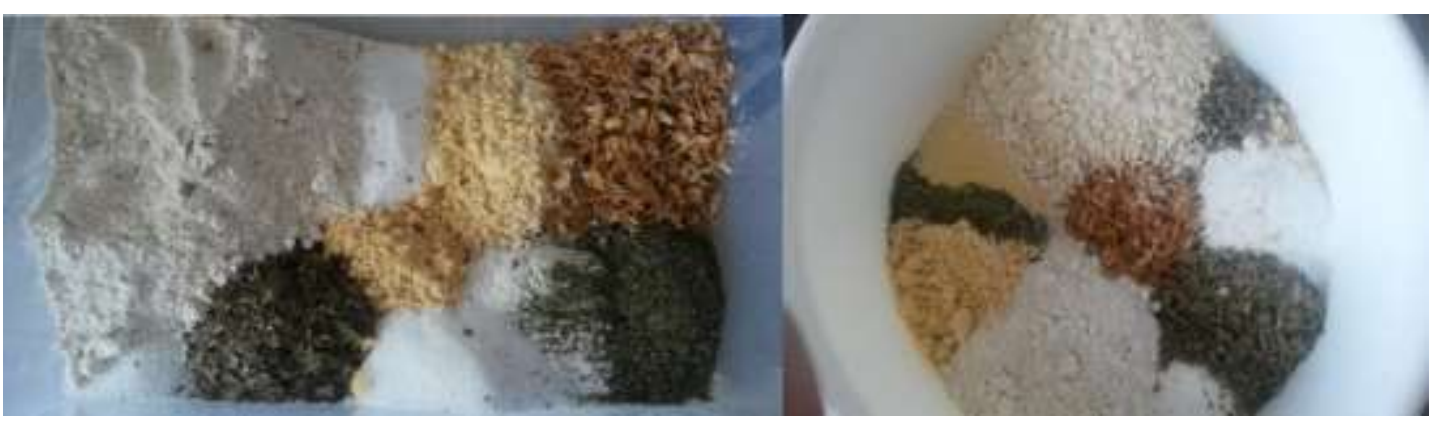

Fonte: Autores.

Uma subamostra das misturas das sopas instantâneas foram armazenadas à temperatura ambiente para posteriores análises microbiológica e análise sensorial. As demais foram congeladas a $-18^{\circ} \mathrm{C}$ para análise da composição centesimal. 


\section{Análises microbiológicas da mistura para sopa instantânea e das farinhas de peixe}

A análise microbiológica da mistura para sopa instantânea foi realizada no Departamento de Análises Clínicas no laboratório de Microbiologia e Microscopia de Alimentos da Universidade Estadual de Maringá - UEM.

As análises microbiológicas foram realizadas para o número mais provável (NMP) de coliformes a $35^{\circ} \mathrm{C}$ e $45^{\circ} \mathrm{C}$, contagem de Staphylococcus coagulase positiva em UFC/grama e de Salmonella spp, de acordo com APHA (1992). O protocolo microbiológico seguiu os padrões recomendados pela Resolução RDC n ${ }^{\circ} 12$, de 2 de janeiro de 2001, da Agência Nacional de Vigilância Sanitária (Brasil, 2001).

\section{Composição centesimal da mistura para sopa instantânea e das farinhas de peixe}

$\mathrm{Na}$ determinação da composição centesimal os teores de umidade, cinzas e extrato etéreo foram padronizados de acordo com a metodologia da Association of Official Analytical Chemists (AOAC, 1995). Os teores de proteína bruta foram avaliados pelo método de semi-micro Kjeldahl (Silva \& Queiroz, 2002). Os teores de carboidratos foram estimados utilizando-se uma fórmula matemática que considera a soma dos valores de umidade, proteína, lipídeos e cinzas subtraídos de 100\% (Brasil, 2003). O valor calórico total foi obtido pela soma da multiplicação dos valores das médias de proteína, lipídios e carboidratos multiplicados pelos fatores 4,9 e 4, respectivamente (Souci et al., 2000).

\section{Análise sensorial da sopa instantânea com as farinhas de peixes}

A análise sensorial foi realizada 24 horas após a elaboração da mistura para sopa instantânea. A preparação das sopas foi realizada por meio da reidratação da mistura para sopa instantânea com água na proporção de 3:1, ou seja, para cada grama de mistura utilizou-se $3 \mathrm{ml}$ de água fervente, preparada em fogão a gás até que a mistura ficasse totalmente homogênea e com aparência, textura e sabor característicos o que demorou aproximadamente 15 minutos. Para a análise sensorial foi fornecido aproximadamente $20 \mathrm{ml}$ de sopa de cada tratamento a 50 provadores que avaliaram as características de aroma, cor, sabor, textura, aparência e aceitação geral. As amostras foram colocadas em pequenos copos plásticos (tipo café) identificadas e oferecidas aos provadores. Estes receberam as amostras codificadas de forma aleatória e uma ficha para avaliação sensorial das diferentes sopas elaboradas. Para tanto, foi utilizada uma ficha com escala hedônica de 9 pontos, tendo como os extremos: 1 (desgostei muitíssimo) e 9 (gostei muitíssimo) (Dutcosky, 2013). Também foi avaliada a intenção de compra. Para essa avaliação utilizou-se a escala de 5 pontos, na qual 5 representava a nota máxima "certamente compraria" e 1 representava a nota mínima "certamente não compraria", empregando os procedimentos descritos para análise sensorial (Meilgaard et al., 1991; Damásio e Silva, 1996).

\section{Delineamento experimental}

O delineamento experimental foi inteiramente casualizado, com quatro tratamentos, sendo a inclusão das diferentes farinhas de tilápia (Trat=1), tilápia defumada (Trat=2), tilápia defumada com salmão (Trat=3) e a tilápia defumada com atum (Trat4) na mistura das sopas instantâneas, com 5 repetições por tratamento.

Os resultados das variáveis analisadas na composição centesimal $(n=5)$ foram submetidos à análise de variância, $\mathrm{e}$ as médias comparadas pelo teste de Tukey, nível de 5\% de probabilidade (Statistical Analysis System - SAS, SAS Inst. Inc. Cary, NC, USA).

Os dados das análises sensoriais foram submetidos ao procedimento UNIVARIATE do SAS, para verificação das pressuposições da análise de variância analisados. Caso as pressuposições não fossem atendidas pelo procedimento GENMOD do SAS foi utilizado gamma com função de ligação inversa. 
Foi considerado o efeito de tratamento e degustadores, testando a inclusão contra o controle, por meio de teste de médias e o comportamento das notas dos degustadores em função do tratamento. As análises microbiológicas foram realizadas apenas para caracterização das farinhas e das misturas das sopas instantâneas, realizando assim uma análise descritiva.

\section{Resultados e Discussão}

De acordo com resultados microbiológicos expressos na Tabela 2, a farinha de peixe de tilápia (FT), de tilápia defumada (FTD), a farinha de tilápia defumada e salmão (FTS) e a farinha de tilápia defumada e atum (FTA), bem como todas as formulações das misturas das sopa com as referidas farinhas (tilápia-SFT; tilápia defumada-SFTD; tilápia com salmão-SFTDS e tilápia com atum-SFTDA), estavam aptos para consumo humano, não sendo detectada a presença de Salmonella sp, estando dentro dos padrões estipulados pela RDC nº12, da Agência Nacional de Vigilância Sanitária do Ministério da Saúde (BRASIL, 2001).

Tabela 2. Análise microbiológica das farinhas de peixe de diferentes espécies e das misturas das sopas instantâneas com as referidas farinhas de peixe.

\begin{tabular}{lcccc}
\hline & \multicolumn{4}{c}{ Análises microbiológicas } \\
\cline { 2 - 5 } Tratamento & $\begin{array}{c}\text { Coliformes a } \\
35^{\circ} \mathrm{C} \\
(\mathrm{NMP} / \mathrm{g})\end{array}$ & $\begin{array}{c}\text { Coliformes a } \\
45^{\circ} \mathrm{C} \\
(\mathrm{NMP} / \mathrm{g})\end{array}$ & $\begin{array}{c}\text { Staphylococcus } \\
\text { coagulase } \\
\text { positiva }(\mathrm{UFC} / \mathrm{g})\end{array}$ & $\begin{array}{c}\text { Salmonella } \\
\mathrm{sp.} 25 \mathrm{~g}\end{array}$ \\
\hline Farinhas & $<3$ & $<3$ & $<10^{2}$ & Ausência \\
\hline FT & $<3$ & $<3$ & $<10^{2}$ & Ausência \\
FTD & $<3$ & $<3$ & $<10^{2}$ & Ausência \\
FTDS & $<3$ & $<3$ & $<10^{2}$ & Ausência \\
FTDA & $<3$ & $<3$ & & Ausência \\
\hline Misturas das sopas com as diferentes farinhas de peixes & $<3$ & $<10^{2}$ & Ausência \\
\hline SFT & $<3$ & $<3$ & $<10^{2}$ & Ausência \\
SFTD & $<3$ & $<3$ & $<10^{2}$ & Ausência \\
SFTDS & $<3$ & & & \\
SFTDA & $<3$
\end{tabular}

$\mathrm{NMP}=$ Números mais provável; $\mathrm{UFC}=$ Unidade formadora de colônia. $\mathrm{FT}=$ farinha de tilápia, $\mathrm{FTD}=$ farinha de tilápia defumada, FTDS= farinha de tilápia defumada com salmão e FTDA= farinha de tilápia defumada com atum; SFT= sopa com farinha de tilápia, SFTD= sopa com farinha de tilápia defumada, SFTDS= sopa com farinha de tilápia com salmão e SFTDA= sopa com farinha de tilápia e atum. Fonte: Autores.

\section{Composição centesimal e valor calórico das farinhas de peixes}

Os valores da composição centesimal e valor calórico das farinhas de peixes estão na Tabela 3. A farinha de tilápia defumada apresentou maior $(\mathrm{P}=0,023)$ teor de umidade comparado as demais farinhas. No entanto, mesmo assim, os valores independem da espécie de peixe foram muito superiores aos teores relatados por Vidal et al. (2011). Os concentrados proteicos obtidos a partir da CMS de resíduos da filetagem de tilápia do Nilo apresentaram, segundo estes autores, entre 0,37\% e 2,11\% de umidade, cuja média foi de $1,38 \%$. 
Tabela 3. Composição centesimal e valor calórico das farinhas de carcaças de diferentes espécies de peixes.

\begin{tabular}{|c|c|c|c|c|c|}
\hline \multirow{2}{*}{ Parâmetros } & \multicolumn{4}{|c|}{ Farinha de peixe } & \multirow[b]{2}{*}{ Valor $\mathrm{P}$} \\
\hline & FT & FTD & FTDS & FTDA & \\
\hline Umidade & $8,36 \pm 0,15^{b}$ & $9,50 \pm 0,17^{\mathrm{a}}$ & $6,50 \pm 0,49^{c}$ & $6,32 \pm 0,08^{c}$ & 0,023 \\
\hline Proteína Bruta & $50,54 \pm 0,8^{b}$ & $48,91 \pm 0,25^{\mathrm{b}}$ & $49,65 \pm 0,51^{\mathrm{b}}$ & $55,88 \pm 0,65^{\mathrm{a}}$ & 0,047 \\
\hline Cinzas & $33,31 \pm 0,07^{\mathrm{a}}$ & $30,05 \pm 0,05^{\mathrm{b}}$ & $32,39 \pm 0,35^{\mathrm{a}}$ & $29,25 \pm 0,22^{\mathrm{b}}$ & 0,010 \\
\hline Lipídeos & $6,07 \pm 0,04^{\mathrm{c}}$ & $10,63 \pm 0,44^{\mathrm{b}}$ & $12,81 \pm 0,05^{\mathrm{a}}$ & $12,85 \pm 0,03^{\mathrm{a}}$ & $<0,0001$ \\
\hline \multicolumn{2}{|c|}{ Valor calórico $(\mathrm{Kcal} / 100 \mathrm{~g}) 263,65 \pm 0,68^{\mathrm{c}}$} & $294,97 \pm 0,15^{\mathrm{b}}$ & $308,47 \pm 3,67^{b}$ & $321,96 \pm 0,35^{\mathrm{a}}$ & 0,0002 \\
\hline
\end{tabular}

*Média na mesma linha seguida de letras distintas diferem entre si pelo teste de Tukey $(\mathrm{P}<0,05)$. ** Valores de carboidratos $<1 \%$. FT= Farinha de tilápia. FTD= Farinha de tilápia defumada. FPTS=Farinha de tilápia com salmão. FPTA=Farinha de tilápia com atum. Fonte: Autores.

Com relação a proteina bruta, o farinha de tilápia defumada com atum (CPTA) apresentou maior percentual de proteína $(55,84 \%)$ que as demais farinhas de peixe, isto pode ser explicado devido à presença da farinha de atum na sua composição, sendo este feito a partir de troncos de atum (maior conteúdo muscular) e não apenas do espinhaço com carne remanescente da filetagem como as demais farinhas elaboradas de peixe (Tabela 3). Todavia, Vidal et al. (2011) encontraram para o concentrado proteico de tilápia a partir da CMS, valores superiores, variando de 59,55\% e 64,35\%, cujo teor médio foi de 62,39\% para esse concentrado proteico. Essa diferença se deve ao tipo de materia prima utilizada, neste trabalho foram as carcaças de duas espécies de peixes nas mesmas condições de filetagem pata elaboração das farinhas, que foi a partir dos espinhaços (coluna vertebral com costelas e carne memanescente da filetagem), enquanto o relatado pro Vital et al. (2011) foi a Carne mecanicamente separada (CMS) do espinhaço da tilápia, portanto, estava presente apenas a carne para elaboração do concentrado proteico.

A farinha de tilápia defumada com salmão (FTDS) e o farinha de tilápia defumada com atum (FTDA) apresentaram maiores $(\mathrm{P}<0,001)$ teores de lipídeos que as demais farinhas, sendo a farinha de tilápia a que apresentou menor teor de lipídeos. Para o teor de cinzas, a FT e FTDS apresentaram as maiores $(\mathrm{P}=0,010)$ porcentagens de cinzas comparado as demais farinhas. Esta explicação também deve-se ao tipo da matéria prima utilizada, pois a quantidade de carne presente no espinhaço dessas epécies foi menor comparada as das demais.

As carcaças sendo defumadas, no momento da prensagem são extraídos menor quantidade de gordura natural comparada as carcaças que foram submetidas ao cozimento e depois prensadas. Com a prensagem é extraída a água e parte da gordura presente na matéria prima. Portanto, os tratamentos que continham farinha de tilápia defumada apresentaram maiores quantidade de lipídeos. Em relação ao valor calórico, os elevados teores de lipídeos presente no farinha de tilápia com atum (CPTA) influenciaram significativamente para que este produto superasse os demais neste quesito. O farinha de tilápia obteve os valores mais baixos para essa variável (Tabela 3).

Esses valores de composição química das farinhas diferem dos obtidos por Petenuci et al. (2010). Os autores relataram valores de $14,2 \%$ de umidade, $40,8 \%$ de proteína, $18,3 \%$ de cinzas e lipídios de 25,3\%. O teores de umidade e lipídeos deste experimento foram inferiores aos relatados por Petenuci et al. (2010), enquanto os teores de proteina bruta e cinzas foram superiores. Também Godoy et al. (2010) avaliarem farinha elaborada a partir de carcaças defumadas de tilápia e encontraram teores de umidade de 17,41\%,32,51\%, de proteína, 19,72\% de extrato etéreo e 26,22\% de cinzas. Esta discrepância entre os valores obtidos pode estar associada tanto com a qualidade da matéria prima, quanto na metodologia aplicada durante a elaboração da farinha de peixe. Pois, os autores em questão não prensaram a matéria prima durante o processamento, aumentando o teor de umidade e de lipídeos. Como as farinhas elaboradas pelos autores anteriormente mencionados apresentavam maior teor de umidade consequentemente os teores de proteina e cinzas são menores.

Souza et al. (2017) elaboraram farinhas de diferentes espécies de peixes, sendo de tilápia, salmão, atum e sardinha. A 
metodologia aplicada foi a de realizar o cozimento das carcaças, prensagem (capacidade de 10 toneladas) moagem, desidratação $(24 \mathrm{~h}$ a $60 \mathrm{oC})$ e nova moagem em moedor tipo faca, para obtenção de uma granulometria fina para as farinhas. Os autores relataram que a umidade variou de $1,78 \%$ a 4,86\%, sendo menor para tilápia e maior para sardinha. O teor de proteina $(83,28 \%)$ foi maior e cinzas $(5,60 \%)$ menor para atum, em função do tipo de resíduo utilizado que continha maior quantidade de carne e menor de ossos (espinhas e coluna vertebral). Já, a farinha de salmão apresentou o menor teor proteico (44,63\%), porém o maior teor de lipídeos $(18,81 \%)$, enquanto, e a farinha de sardinha o menor $(3,998 \%)$. Quanto as cinzas a farinha de tilápia apresentou o maior teor (37,66\%) em relação as demais. Estes valores relatados por Souza et al. (2017) foram muito diferentes dos obtidos neste trabalho, pois a variação dos nutrientes apresentados pelos autores foram muito grandes para a proteina $(44,63 \%$ a $83,28 \%)$, lipídeos (3,98\% a 18,81\%), cinzas $(5,31 \%$ a 37,66\%), sendo o teor de umidade a menor variação comparada aos demais nurientes $(1,78 \%$ a $4,86 \%)$. Mostrando que os fatores ligados ao tipo de materia prima influenciaram muito na composição quimica das farinhas, tanto a espécie quanto ao tipo de parte utilizada para elaboração da farinha, visto que a de atum foi utilizado a parte terminal da coluna vertebral, proximo a nadadeira caudal (contendo maior quantidade de carne).

Portanto, através dos resultados deste trabalho e comparando com os obtidos na literatura, fica claro que a metodologia empregada na produção da farinha de peixe interfere diretamente na qualidade do produto final, assim com a espécie de peixe e forma em que se encontra a materia prima para ser utilizada na elaboração da farinha.

\section{Análise de composição centesimal da mistura da sopa instantânea com farinhas de peixes}

Os resultados da análise centesimal da mistura da sopa instantânea estão na Tabela 4, mostrando que não houve diferença significativa para o teor de proteína e de carboidratos entre os tratamentos, cujos valores médios foram 11,24\% e 49,39\%, respectivamente. A mistura da sopa instanânea com inclusão de FT apresentou maior $(\mathrm{P}=0,006)$ teor de umidade $(23,24 \%) \mathrm{em}$ relação as demais sopas. O percentual de umidade do presente estudo também foi superior ao observado em sopa de peixe elaborada a partir de CMS de piranha-preta (10,53\%) seca em estufa com circulação forçada de ar a $60{ }^{\circ} \mathrm{C}$ por 18 horas (Silva et al., 2003). Tudo indica que após a mistura dos ingredientes, a mistura teria que ter sido submetida a uma desidratação por algumas horas, para que o teor de umidade fosse em torno dos 10\%. Quanto aos lipídeos as misturas para a sopa com FTD e FTDS apresentaram os maiores $(\mathrm{P}<0,001)$ teores $(6,90 \%$ e $6,89 \%$, respectivamente) em relação aos demais tratamentos. Esta característica, de certa forma influenciou no valor calórico, justificando o menor $(\mathrm{P}=0,018)$ valor para a mistura da sopa instantânea com a FT (291,02 Kcal/100g, enquanto as com FTDS e FTDA o valor médio foi de 303,82 Kcal/100g (Tabela 4). A SFTDA apresentou o menor teor de cinzas (9,84\%), apesar de não terem diferido da SFTDS (10,21\%), esses valores mais baixos $(\mathrm{P}=0,01)$ de cinzas fazem inferencia as caracteríticas das matérias primas utilizadas que continham menor quantidade de espinhas na carcaça ou menor percentual de matéria óssea. 
Tabela 4. Composição centesimal de sopa instantânea com inclusão do farinha de tilápia (SFT), farinha de tilápia defumada (SFTD), farinha de tilápia com salmão (SFTDS) e farinha de tilápia com atum (SFTDA).

\begin{tabular}{lccccc}
\hline \multirow{2}{*}{ Parâmetros } & \multicolumn{4}{c}{ Tratamentos } & \\
\cline { 2 - 5 } & \multicolumn{1}{c}{ SFT } & SFTD & SFTDS & SFTDA & 0,006 \\
\hline Umidade & $23,24 \pm 0,45^{\mathrm{a}}$ & $22,57 \pm 0,02^{\mathrm{b}}$ & $22,40 \pm 0,21^{\mathrm{b}}$ & $22,42 \pm 0,12^{\mathrm{b}}$ & 0,317 \\
Proteína Bruta & $11,13 \pm 0,07$ & $11,11 \pm 0,09$ & $11,17 \pm 0,04$ & $11,57 \pm 0,23$ & 0,01 \\
Cinzas & $10,66 \pm 0,26^{\mathrm{a}}$ & $10,44 \pm 0,11^{\mathrm{a}}$ & $10,21 \pm 0,04^{\mathrm{ab}}$ & $9,84 \pm 0,31^{\mathrm{b}}$ & $<0,001$ \\
Lipídeos & $5,33 \pm 0,88^{\mathrm{b}}$ & $6,90 \pm 0,22^{\mathrm{a}}$ & $6,89 \pm 0,22^{\mathrm{a}}$ & $6,53 \pm 0,03^{\mathrm{b}}$ & 0,073 \\
Carboidratos & $49,63 \pm 0,00$ & $48,97 \pm 0,46$ & $49,32 \pm 0,21$ & $49,64 \pm 0,01$ & 0,018 \\
\hline Valor calórico $(\mathrm{Kcal} / 100 \mathrm{~g})$ & $291,02 \pm 8,71^{\mathrm{c}}$ & $302,41 \pm 0,65^{\mathrm{b}}$ & $303,63 \pm 0,20^{\mathrm{a}}$ & $304,00 \pm 0,46^{\mathrm{a}}$ & 0 \\
\hline
\end{tabular}

Dados expressos em média \pm desvio padrão. $5^{*}$ Médias na mesma linha seguidas de letras distintas diferem entre si pelo teste de Tukey (5\%). Fonte: Autores.

Caballero-Córdoba et al. (1994), trabalhando com sopa cremosa semi- instantânea a base de farinhas de trigo e soja desengordura com cenoura e chuchu, obtiveram valores de umidade inferiores (variou de 1,70\% a 3,00\%) aos obtidos neste experimento (Tabela 4). Também para proteina variou de $15,56 \%$ a $34,75 \%$, lipídeos de $0,71 \%$ a $0,93 \%$ e cinzas de $1,77 \%$ a $3,88 \%$ teores muito inferiores aos observados na sopa instantânea com as farinhas de peixes. Portanto, quando adicionado um farinha de peixe numa mistura para sopa instantânea é esperado que o teor nutritivo desta seja muito superior a qualquer outra mistura sem a presença de farinha de origem animal de qualquer espécie. Dessa forma, mostra a importância da inclusão de uma proteina de origem animal nesse tipo de produto, pois além de aumentar o teor proteico, aumentou a quantidade de matéria mineral, consequente determinados minerais importantes, tais como o cálcio, fósforo, ferro, assim como a quantidade e qualidade de lipídeos nessa sopa. De acordo com Souza et al. (2017), farinhas de peixes de diferentes espécies apresentam 0,90\% e 0,91\% (atum) e 9,39\% e 6,08\% (tilápia), respectivamente de cálcio e fósforo. O teor de ferro foi superior para a farinha de sardinha $(121,95 \mathrm{mg} / \mathrm{kg})$ e o menor para farinha de tilápia $(35,18 \mathrm{mg} / \mathrm{kg})$. Portanto, pode-se inferir que nessas sopas elaboradas também deve ter aumentando os níveis de minerais, apesar dessa análise não ter sido realizada. Também, a questão dos ácidos graxos, sabe-se que os peixes marinhos apresentam ômega 3, sendo muito superior aos peixes de água doce (Souza et al., 2017). Sendo assim, para as sopas com a inclusão da farinha de salmão e de atum na mistura, deve ter elevado os níveis desse ácido graxo nessa mistura, melhorando consequentemente o valor nutriconal da mesma. Visto que Souza et al. (2017) obtiveram para as farinhas avaliadas de salmão $53,71 \mathrm{~g} / \mathrm{kg}$, de sardinha $47,46 \mathrm{~g} / \mathrm{kg}$ e de atum $136,98 \mathrm{~g} / \mathrm{kg}$ de ômega-3. Os mesmos autores mencionam que as referidas farinhas apresentam como aminoácidos essenciais majoritários o ácido glutâmico, fenilalanica+tirosina, lisina, leucina, glicina e ácido aspártico. Com isso, Souza et al. (2017) afirmam que todas as farinhas avaliadas das quatro esécies de peixes apresentam elevado valor biológico e nutricional, além da boa fonte de cálcio, fósforo e ferro. Concluindo-se que o mesmo é esperado para essas misturas das sopas intantâneas, pois constam tais farinhas de peixes (tilápia, salmão e atum) na sua formulação (Tabela 1).

\section{Análise sensorial das sopas instantânea com inclusão de farinhas de peixes}

Em relação às características sensoriais, não houve diferença $(\mathrm{P}>0,05)$ significativa para cor, aroma e textura, cujas médias foram 6,78,6,57 e 6,81, respectivamente. O sabor foi melhor $(\mathrm{P}<0,001)$ para sopa com a inclusão da farinha de tilápia e sopa com a farinha de tilápia defumada em relação aos demais tratamentos. Já, para impressão global a farinha de tilápia foi superior $(\mathrm{P}<0,001)$ a sopa de farinha de salmão e atum, entretanto, não diferiu da sopa de farinha de tilápia defumada. De acordo com os resultados de intenção de compra dos provadores, a maioria possivelmente compraria a mistura de sopa instantânea com 
tilápia, com tilápia defumada e tilápia defumada com salmão (Tabela 5). Mas com a inclusão do atum teria dúvidas (talvez compraria e talvez não compraria).

Tabela 5. Análise sensorial de sopa instantânea com inclusão de farinha de tilápia (SFT), farinha de tilapia defumada (SFTD), farinha de tilápia com salmão (SFTDS) e farinha de tilapia com atum (SFTDA). Dados expressos em média \pm desvio padrão.

\begin{tabular}{|c|c|c|c|c|c|}
\hline \multirow{2}{*}{ Parâmetros } & \multicolumn{4}{|c|}{ Tratamentos } & \multirow{2}{*}{ Valor P } \\
\hline & $\begin{array}{l}\text { SFT } \\
\end{array}$ & SFTD & SFTDS & SFTDA & \\
\hline \multicolumn{6}{|l|}{ Atributos* } \\
\hline Cor & $6,88 \pm 0,04$ & $6,90 \pm 0,06$ & $6,78 \pm 0,02$ & $6,56 \pm 0,17$ & 0,706 \\
\hline Aroma & $6,64 \pm 0,04$ & $6,54 \pm 0,02$ & $6,58 \pm 0,00$ & $6,52 \pm 0,03$ & 0,987 \\
\hline Textura & $7,14 \pm 0,23$ & $6,94 \pm 0,09$ & $6,84 \pm 0,02$ & $6,34 \pm 0,33$ & 0,086 \\
\hline Sabor & $7,46 \pm 0,71^{\mathrm{a}}$ & $7,12 \pm 0,47^{\mathrm{a}}$ & $6,04 \pm 0,29^{b}$ & $5,20 \pm 0,88^{\mathrm{b}}$ & $<0,001$ \\
\hline Imp. Global & $7,28 \pm 0,55^{\mathrm{a}}$ & $7,00 \pm 0,35^{\mathrm{ab}}$ & $6,20 \pm 0,21^{\mathrm{bc}}$ & $5,52 \pm 0,69^{c}$ & $<0,001$ \\
\hline Intenção de compra** & $3,84 \pm 0,27^{\mathrm{a}}$ & $3,66 \pm 0,14^{\mathrm{a}}$ & $3,32 \pm 0,09^{\mathrm{ab}}$ & $3,02 \pm 0,31^{\mathrm{b}}$ & 0,004 \\
\hline
\end{tabular}

Médias na mesma linha seguidas de letras distintas diferem entre si pelo teste de Tukey $(\mathrm{P}<0,05)$. $*$ Escala hedônica 9 pontos. **Escala hedônica 5 pontos. Fonte: Autores.

Produtos como a sopa instantânea são relativamente novos no mercado e não existem muitos estudos com enfoque no enriquecimento nutricional deste tipo de produto. O que se encontra seria em termos de mais uma opção para consumir um produto rápido no preparo, facilitando com isso a vida do consumidor. Contudo, Caballero-Córdoba et al. (1994), trabalhando com sopa cremosa semi-instantânea a base de farinhas de trigo e soja desengordura com cenoura e chuchu, obtiveram valores superiores ao presente estudo para as características sensoriais, cujas notas para a cor, aroma, textura e sabor foram entre 7 e 8 (gostei moderadamente/gostei muito). O sabor de peixe difere do tradicionalmente consumido pela população, principalmente para uma sopa, suas características e aceitabilidade como ingredientes utilizadas em sopa e demais produtos, ainda é uma ideia nova, havendo necessidade de mais trabalhos com inclusão de peixe em sopas ou em outros produtos alimentícios. Mas, do ponto de vista nutricional, pode-se observar que é uma excelente alternativa em especial para crianças, idosos e convalescentes, em função do alto valor biológica da sopa, devido ao teor proteico e os aminoácidos essenciais presentes, enriquecida por ômega-3 e 6, bem como os minerais.

\section{Conclusão}

Considerando a boa qualidade nutricional e microbiológica das farinhas elaboradas e das misturas das sopas instantâneas, além da boa aceitação pelos consumidores é uma boa alternativa para ter esse tipo de produto no comércio. Contudo, a sopa com inclusão de farinha de tilápia foi considerada a mais saborosa, e a inclusão da farinha de atum e de salmão intensificou o aroma da sopa, diminuindo a aceitação deste produto, em especial a com atum. Mas, seria interessante a realização de novos estudos para melhorar o aroma desta mistura de sopa instantânea, visto que na literatura mostra que essa farinha apresenta elevado teor de omega-3. Outro aspecto inportante a ser considerado é quanto a quantidade de resíduos que normalmente não tem um destino nobre e com a produção ds farinhas e inclusão nas sopas ou outro produto alimentício é uma forma de agregar valor à cadeia produtiva do peixe, além de evitar impacto ambiental. 


\section{Referências}

AOAC. (1995). Oficial Methods of Analysis of the Association of Official Analytical Chemists. Arlington, International.

APHA. (1992). Americam Pubblic Health Association. Compendium of methods for the microbiological examination of foods. (3a ed.), 15 APHA.

Bligh, E. G., \& Dyer, W. J. (1959). A rapid method of total lipid extraction and purification. Canadian Journal of Biochemistry and Physiology, 37, 911-17.

Brasil. (2001). ANVISA - Agência Nacional de Vigilância Sanitária. Resolução n ${ }^{\circ}$ 90, de 18 de outubro de 2001 - Regulamento Técnico para Fixação de Identidade e Qualidade de Pão. Diário Oficial da República Federativa do Brasil, Brasília, 18 de outubro de 2001.

Brasil. (2003). ANVISA - Agência Nacional de Vigilância Sanitária. RDC nº 360, de 23 de dezembro de 2003. Aprova o regulamento técnico sobre rotulagem nutricional de alimentos embalados. Diário Oficial da República Federativa do Brasil, Brasília, 23 dez. de 2003.

Caballero-Córdoba, G. M., Wang, S., \& Sgarbieri, V. C. (1994). Caracteristicas Nutricionais e Sensoriais de Sopa Cremosa Semi-Instantânea à Base de Farinhas de Trigo e Soja Desengordurada. Pesquisa Agropecuária Brasileira, 29, 1137-2243.

Coradini, M. F., Souza, M. L. R., Verdi, R., Goes, E. S. D. R., Kimura, K. S., \& Gasparino, E. (2015). Quality evaluation of onion biscuits with aromatized fishmeal from the carcasses of the Nile tilapia. Boletim do Instituto de Pesca, 41, 719-728.

Damásio, M. H., \& Silva, M. A. A. P. (1996). Curso de treinamento em análise sensorial. Apostila Campinas: Fundação Tropical de Tecnologia "André Tosello". Dutcosky, S. D. (2013). Análise sensorial de alimentos. 4. ed. Curitiba: Champagnat.

Godoy, L. C., Franco, M. L. R. S., Franco, N. P., Silva, A. F., Assis, M. F., Souza, N. E., Matsushita, M., \& Visentainer, J. V. (2010). Análise sensorial de caldos e canjas elaborados com farinha de carcaças de peixe defumadas: Aplicação na merenda escolar. Ciência e Tecnologia de Alimentos, 30 , p. 86-89.

Godoy, L. C., Franco, M. L. R. D. S., De Souza, N. E., Stevanato, F. B., \& Visentainer, J. V. (2013). Development, preservation, and chemical and fatty acid profiles of Nile tilapia carcass meal for human feeding. Journal of Food Processing and Preservation, 37(2), 93-99.

Islam, M., Sarker, M. N. I., Islam, M. S., Prabakusuma, A. S., Mahmud, N., Fang, Y., \& Xia, W. (2018). Development and quality analysis of protein enriched instant soup mix. Food and Nutrition Sciences, 9(6), 663-675.

Justen, A. P., Souza, M. L. R. D., Monteiro, A. R., Mikcha, J. M., Gasparino, E., Delbem, Á. B., \& Del Vesco, A. P. (2017). Preparation of extruded snacks with flavored flour obtained from the carcasses of Nile tilapia: physicochemical, sensory, and microbiological analysis. Journal of Aquatic Food Product Technology, 26(3), 258-266.

Karthika Devi, B., Kuriakose, S. P., Krishnan, A. V. C., Choudhary, P. \& Rawson, A. (2016). Utilization of By-Product from Tomato Processing Industry for the Development of New Product. Journal of Food Processing \& Technology, 7, 608.

Li, W., \& Savage, G. P. (2015). Oxalate Content of the Herb Good-King-Henry, Blitum Bonus-Henricus. Foods, $4,140-147$.

Matiucci, M. A., Souza, M. L. R. D., Chambo, A. P. S., Vitorino, K. C., Réia, S., \& Verdi, R. (2019). Patês elaborados a partir de resíduos do beneficiamento de tilápia com e sem defumação. Iniciação Científica Cesumar, 21, 163-173.

Meilgaard, M., Civile, G. V., \& Carr, B. T. Sensory evaluation techniques. (2a ed.), Boca Raton: CRC Press, Inc., 1991.

Monteiro, M. L. G., Mársico, E. T., Lázaro, C. A., Ribeiro, R. O., Jesus, R. S., \& Conte-Júnior, C. A. (2014). Flours and instant soup from tilapia wastes as healthy alternatives to the food industry. Food Science and Technology Research, 20(3), 571-581.

Petenuci, M. E., Stevanato, F. B., Morais, D. R. D., Santos, L. P., Souza, N. E. D., \& Visentainer, J. V. (2010). Composição e estabilidade lipídica da farinha de espinhaço de tilapia. Ciência e Agrotecnologia, 34(5), 1279-1284.

Silva, D. J., \& Queiroz, A. C. Análise De Alimentos: Métodos Químicos e Biológicos. (3a ed.), Universidade Federal de Viçosa, 2002. 235p.

Silva, M. A. B., Lessi, E., Jesus, R. S., \& Falcão, P. T. (2003). Utilização de piranha-preta Serrasalmus rhombeus para elaboração de sopas de peixe. In: Congresso Brasileiro de Engenharia de Pesca, 13., 2003, Porto Seguro/BA. Resumos. Porto Seguro: AEP-BA, 2003. p. 1122.

Škaljac, S., Petrović, L., Tasić, T., Ikonić, P., Jokanović, M., Tomović, V., \& Škrbić, B. (2014). Influence of smoking in traditional and industrial conditions on polycyclic aromatic hydrocarbons content in dry fermented sausages (Petrovská klobása) from Serbia. Food Control, 40, 12-18.

Souci, S. W., Fachman, H., \& Kraut, E. (2000). Foods Composition and Nutrition Tables. Medpharm Scientific Publishers. (6a ed.),

Souza, M. L. R., Yoshida, G. M., Campelo, D. A. V., Moura, L. B., Xavier, T. O., \& Goes, E. S. R. (2017). Formulation of fish waste meal for human nutrition. Acta Scientiarum Technology, 39, 525-531.

Sudarsan, S. M., Santhanam, S. G. \& Visalachi, V. (2017) Development and Formulation of Instant Soup Mix from Sprouted Horse Gram and Radish Leaves. International Journal of Home Science, 3, 346-349.

Tilami, S. K., \& Sampels, S. (2018). Nutritional value of fish: lipids, proteins, vitamins, and minerals. Reviews in Fisheries Science \& Aquaculture, 26(2), 243253.

Vidal, J. M. A., Rodrigues, M. D. C. P., Zapata, J. F. F., \& Vieira, J. M. M. (2011). Concentrado protéico de resíduos da filetagem de tilápia-do-nilo (Oreochromis niloticus): caracterização físico-química e aceitação sensorial. Revista Ciência Agronômica, 42(1), 92-99. 